\title{
Linfohistiocitosis hemofagocítica secundaria/ síndrome de liberación de citoquinas por SARS-CoV-2: una revisión concisa
}

Secondary hemophagocytic lymphohystiocytosis/cytokines release syndrome in SARS-CoV-2: a concise review Linfohistiocitose hemofagocítica secundária/síndrome de liberação de citocina SARS-CoV-2: uma revisão concisa

Mario Andrés Hernández-Sómerson, ${ }^{\star, \neq}$ Omar Bernal-Ramírez, ${ }^{\S}$ Juan Camilo Jiménez-Palomino, ${ }^{\S}$ Samuel Rodríguez-Urueña, ${ }^{\S}$ Fernando Montoya Agudelo ${ }^{\ddagger}$

\section{RESUMEN}

Hay evidencia creciente de una desregulación del sistema inmune asociada a la infección por SARS-CoV-2, que se relaciona directamente con la severidad y la mortalidad por COVID-19. Niveles elevados de interleucinas, en especial IL-6, IL-1 y factor de necrosis tumoral alfa, disminuyen la actividad antiviral de interferón gamma e inducen un descenso en el recuento de linfocitos T y un estado de agotamiento inmune que lleva al deterioro clínico observado en estos pacientes. Este evento fisiopatológico ha sido llamado tormenta de citoquinas y resulta común a otras entidades como la linfohistiocitosis hemofagocítica secundaria y el síndrome de activación macrofágica, visto en enfermedades autoinmunes. No hay criterios diagnósticos específicos para este síndrome de liberación de citoquinas asociado con COVID-19. Estos hallazgos abren la puerta a la utilización de tratamiento inmunomodulador, en especial a la terapia dirigida contra citoquinas, pero se requieren estudios clínicos que establezcan claramente la relación de riesgo y beneficio.

Palabras clave: COVID-19, tormenta de citoquinas, linfohistiocitosis hemofagocítica, neumonía, SARS-CoV-2.

\section{ABSTRACT}

There is increasing evidence of a dysregulation of the immune system associated with SARS-CoV-2 infection, which is directly related to COVID-19 severity and mortality. High levels of interleukins, especially IL-6, IL-1 and TNF alpha, decrease the anti-viral activity of interferon gamma and induce a decrease in the Tlymphocyte count and a state of immune exhaustion that is associated with the clinical deterioration observed in these patients. This pathophysiological event has been called a cytokine storm and is common to other entities such as secondary hemophagocytic lymphohistiocytosis and macrophage activation syndrome seen in autoimmune diseases. There are no specific diagnostic criteria for this cytokine release syndrome associated with COVID-19. All these findings open the door to the use of immunodulatory treatment, especially therapy directed against cytokines, but clinical studies are required that clearly establish the risk-benefit ratio.

Keywords: COVID-19, cytokine storm, hemophagocytic lymphohystiocytosis, pneumonia, SARS-CoV-2.

\section{RESUMO}

Há evidências crescentes de uma desregulação do sistema imunológico associada à infecção por SARS-CoV-2, que está diretamente relacionada à gravidade e mortalidade de COVID-19. Altos níveis de interleucinas, especialmente IL-6, IL-1 e fator de necrose tumoral alfa, diminuem a atividade antiviral do interferon gama e induzem uma diminuição na contagem de linfócitos $T$ e um estado de depleção imunológica que leva à deterioração clínica. observado nesses pacientes. Esse evento fisiopatológico fo denominado tempestade de citocinas e é comum a outras entidades, como a linfo-histiocitose hemofagocítica secundária e a síndrome de ativação de macrófagos, observadas em doenças autoimunes. Não há critérios de diagnóstico específicos para essa síndrome de liberação de citocinas associada

\footnotetext{
* Méderi - Hospital Universitario Mayor. Universidad del Rosario. Bogotá, Colombia. ‡ Clínica de la Mujer. Bogotá, Colombia.

$\S$ Clínica CAFAM. Bogotá, Colombia.
}

\section{Recibido: 20/05/2020. Aceptado: 23/12/2020.}

Citar como: Hernández-Sómerson MA, Bernal-Ramírez O, Jiménez-Palomino JC Rodríguez-Urueña S, Montoya AF. Linfohistiocitosis hemofagocítica secundaria/síndrome de liberación de citoquinas por SARS-CoV-2: una revisión concisa. Med Crit. 2021;35(5):263-268. https://dx.doi.org/10.35366/102355

www.medigraphic.com/medicinacritica ao COVID-19. Esses achados abrem as portas para o uso de tratamento imunodulatório, especialmente terapia direcionada contra citocinas, mas são necessários estudos clínicos que estabeleçam claramente a relação. risco e benefício.

Palavras-chave: COVID-19, tempestade de citocinas, linfohistiocitose hemofagocítica, pneumonia, SARS-CoV-2.

\section{INTRODUCCIÓN}

La linfohistiocitosis hemofagocítica (LHH) es un síndrome caracterizado por la desregulación de la respuesta inmune, en cuyo eje central se encuentra la expresión exagerada de citoquinas proinflamatorias que llevan a disfunción orgánica múltiple y a altas tasas de mortalidad. ${ }^{1}$ Se considera una entidad rara, con una incidencia de 1 por cada 800,000 personas por año en la población general, edad media de presentación a los 50 años y proporción hombre-mujer de 1:7.2

Clásicamente se han reportado dos formas de presentación: una primaria o familiar, descrita principalmente en la infancia, ocasionada por defectos genéticos específicos en las vías de citotoxicidad de las células natural killer (NK) y linfocitos $\mathrm{T}$, que siguen una herencia autosómica recesiva. ${ }^{3} \mathrm{La}$ forma secundaria o adquirida es la más frecuente en los adultos, se asocia a infecciones virales, tales como virus Epstein Barr, citomegalovirus y virus de inmunodeficiencia humana, pero también a una variedad de bacterias, hongos y parásitos que actúan como disparadores. De igual manera, existe asociación fuerte a neoplasias hematológicas como el linfoma de células NK o de células T (35\%), linfomas de células B (32\%) y leucemias (6\%). ${ }^{4}$ Un subgrupo de pacientes van a expresar este síndrome en el contexto de enfermedades autoinmunes, tales como enfermedad de Still, lupus y vasculitis; en ellos se aconseja utilizar el término síndrome de activación macrofágica, dado que su tratamiento puede ser diferente a otras formas de presentación. ${ }^{2}$

Durante la pandemia por SARS-CoV-2, causante de la enfermedad por coronavirus del 2019 (COVID-19), se han reportado varios hallazgos con respecto a la disfunción del sistema inmunológico que se relacionan directamente con la severidad de los casos y predicen alto riesgo de muerte; ${ }^{5,6}$ entre ellos, un nivel excesivo de citoquinas proinflamatorias y quimiocinas, descritas 
como tormenta de citoquinas, que llevan a síndrome de dificultad respiratoria aguda y disfunción en otros órganos. Además, se han documentado niveles bajos de linfocitos T CD4+, CD8+ y neutrófilos, fiebre persistente y elevación de ferritina. ${ }^{7,8}$ Estas alteraciones en conjunto rememoran el síndrome de activación macrofágica visto en enfermedades autoinmunes y a los cuadros de LHH secundaria, compartiendo una fisiopatología que se ha agrupado bajo el título de síndrome de liberación de citoquinas asociado a enfermedad por coronavirus 2019 (COVID-19). Algunos autores ${ }^{9,10}$ recomiendan la utilización de los criterios diagnósticos de LHH para su temprana identificación y explorar el manejo inmunomodulador precoz con el fin de impactar la mortalidad.

Este artículo revisa las características clínicas y bioquímicas de la tormenta de citoquinas en la infección por SARS-CoV-2, los desenlaces clínicos asociados a su presencia y un acercamiento a los potenciales blancos terapéuticos.

\section{MATERIAL Y MÉTODOS}

Se realizó una revisión narrativa con el objetivo de identificar información respecto a la presencia del síndrome de liberación de citoquinas en la infección por SARSCoV-2, específicamente literatura relevante en el diagnóstico, la relación con la severidad por COVID-19 y el tratamiento. La búsqueda fue realizada en las bases de datos: PubMed, Embase, MEDLINE y CENTRAL; se tuvieron en cuenta artículos originales, de revisión y consensos nacionales e internacionales sobre el tema, sin restricción de idiomas, publicados hasta el 18 de mayo de 2020. Se evaluaron en total 112 artículos y se escogieron 46 para elaborar el manuscrito, el cual fue aprobado por cada uno de los autores. Por tratarse de una revisión narrativa de la literatura, no fue necesaria la aprobación por el comité de ética.

\section{DIAGNÓSTICO}

No hay características clínicas que puedan distinguir entre COVID-19 y la infección por otros virus respiratorios. La presencia de fiebre se observa en $99 \%$ de los pacientes, fatiga en $70 \%$ y tos seca en $59 \% .{ }^{11,12}$ La disnea suele aparecer luego del quinto día de inicio de los síntomas, y el viraje a la severidad se da luego del séptimo día, habitualmente con aumento en la disnea y progresión a síndrome de dificultad respiratoria aguda (SDRA). ${ }^{11-13}$

Se debe sospechar el síndrome de liberación de citoquinas (SLC), si luego del día cinco de inicio de los síntomas, hay fiebre persistente, citopenias (sobre todo linfopenia) o disfunción de órganos, en especial el pulmón que será el más frecuentemente afectado. Estos hallazgos asociados con la elevación de marcadores inflamatorios, tales como ferritina, proteína $\mathrm{C}$ reactiva, lactato deshidrogenasa, dímero $\mathrm{D}$ e interleucinas, predicen el compromiso multiorgánico y aumentan la probabilidad de muerte. ${ }^{5,14}$

No existen criterios diagnósticos para el SLC en COVID-19. Dadas las características similares con la linfohistiocitosis hemofagocítica secundaria, varios autores ${ }^{9,10}$ proponen la utilización de sus criterios diagnósticos para hacer un reconocimiento temprano mediante el uso de las clasificaciones LHH de $2004^{15}$ y HScore de 2014 (Tabla 1). ${ }^{16}$ La primera ha sido validada en población pediátrica e incluye criterios genéticos y pruebas difíciles de conseguir en nuestro medio, como la actividad de las células NK, por lo cual la consideramos de más difícil aplicación. El HScore fue diseñado para formas adquiridas de $\mathrm{LHH}$, cada ítem tiene un valor y la suma total de los valores arroja un puntaje final entre 90 y 250. El punto de corte óptimo fue de 169, el cual clasifica adecuadamente $90 \%$ de los casos de LHH. ${ }^{16}$ Muchos de estos puntos son encontrados en las personas con COVID-19 severo (Tabla 2). Sin embargo, en estas dos clasificaciones ninguno de los criterios es específico de LHH, a excepción de los niveles de CD25 soluble, los cuales no están ampliamente disponibles. Además, la hemofagocitosis en médula ósea, por ejemplo, puede estar presente en pacientes sin LHH, como en procesos sépticos, falla multiorgánica o posterior a transfusión de glóbulos rojos. ${ }^{2}$

En niños con artritis idiopática juvenil sistémica, la relación ferritina-velocidad de sedimentación globular resultó ser una herramienta útil para el diagnóstico del síndrome de activación macrofágica ${ }^{17}$ y valdría la pena realizar estudios en COVID-19 severo para determinar un punto de corte adecuado que diagnostique la presencia de SLC.

\section{Tormenta de citoquinas como predictor de severidad}

Cerca de $20 \%$ de los casos de COVID-19 van a requerir hospitalización y, de ellos, entre 5-15\% ingresarán a

Tabla 1: HScore para diagnóstico de linfohistiocitosis hemofagocítica.

\begin{tabular}{ll}
\hline Inmunosupresión conocida & 0 (no) 018 (sí) \\
Temperatura & $0\left(<38.4^{\circ}\right), 33\left(38.4^{\circ}-39.4^{\circ}\right), 49\left(>39.4^{\circ}\right)$ \\
Citopenias & $0(1$ linaje), 24 (2 linajes), 34 (3 linajes) \\
Leucocitos $<5,000 / \mathrm{mm}^{3}$ & \\
Plaquetas $<110,000 / \mathrm{mm}^{3}$ & \\
$\mathrm{Hg}<9.2 \mathrm{~g} / \mathrm{dL}$ & \\
Ferritina $(\mathrm{ng} / \mathrm{mL})$ & $0(<2,000), 35(2,000-6,000), 50(>6,000)$ \\
Triglicéridos $(\mathrm{mg} / \mathrm{dL})$ & $0(<132), 44(132-353), 64(>353)$ \\
Fibrinógeno $(\mathrm{g} / \mathrm{L})$ & $0(>2.5), 30(<2.5)$ \\
GOT (UI/L) & $0(<30) 019(>30)$ \\
Hemofagocitosis en médula ósea & $0(\mathrm{no}) 035(\mathrm{si})$ \\
\hline
\end{tabular}

GOT = transaminasa glutámico oxalacética.

Adaptada de: Fardet $L$, et al. ${ }^{16}$ 
Tabla 2: Características de linfohistiocitosis hemofagocítica en COVID-19.

\begin{tabular}{cc}
\hline HScore & COVID-19 \\
\hline Fiebre & Presente \\
Organomegalia & Desconocido \\
Anemia & Presente \\
Trombocitopenia & Presente \\
Leucopenia & Presente \\
Hipertrigliceridemia & Desconocido \\
Fibrinógeno disminuido & Presente \\
Hemofagocitosis & Desconocido \\
Elevación GOT & Presente \\
Inmunosupresión & Posible \\
\hline
\end{tabular}

GOT = transaminasa glutámico oxalacética.

la Unidad de Cuidados Intensivos (UCI) con tasas de mortalidad que fluctúan entre $1-6 \%$ de todos los infectados. ${ }^{7,11,13}$ El tiempo entre el inicio de los síntomas y la hospitalización está alrededor de $9.7 \pm 4.3$ días y la muerte se produce en dos picos, uno en el día 14 y otro alrededor del día 22. ${ }^{7}$ Varios predictores de mortalidad han sido identificados y los más comunes en todas las cohortes han sido: la edad mayor a 60 años, la presencia de comorbilidades como hipertensión, diabetes, insuficiencia cardiaca, cáncer, inmunosupresión, entre otras. También se incluyen la injuria cardiaca y pulmonar, así como marcadores de hiperinflamación tales como linfopenia, niveles elevados de proteína $\mathrm{C}$ reactiva, lactato deshidrogenasa, ferritina, dímero $D$ e interleucinas 1 (IL-1), 6 (IL-6), 10 (IL-10) y factor de necrosis tumoral alfa (FNT- $\alpha)^{5-7,13}$ Estas alteraciones sugieren en conjunto desregulación del sistema inmunológico y tormenta de citoquinas, similar a la reportada en infección por coronavirus asociado a síndrome respiratorio agudo grave (SARS-CoV, por sus siglas en inglés) ${ }^{18} \mathrm{y}$ al síndrome respiratorio de oriente medio (MERS-CoV, por sus siglas en inglés), ${ }^{19}$ que predicen compromiso pulmonar extenso, requerimiento de cuidado intensivo y muerte, de manera independiente al pico de viremia. Pero los betacoronavirus no son los únicos capaces de inducir esta liberación de citoquinas, en la pandemia por virus de la influenza A H1N1 de 2009 se documentaron iguales hallazgos en los pacientes ingresados a las unidades de cuidados intensivos. ${ }^{20}$

Varios estudios retrospectivos han demostrado una asociación directa entre los niveles elevados de ciertas citoquinas y la severidad por COVID-19. Chen y colaboradores ${ }^{8}$ estudiaron 21 pacientes diagnosticados con COVID-19, 11 de ellos con infección severa y 10 con infección moderada, definida por los criterios de las guías chinas para el diagnóstico y tratamiento de COVID-19 (sexta edición). ${ }^{21}$ En los casos severos se observó una disminución significativa en los niveles de linfocitos T CD4+ y CD8+ $(p=0.018,0.035$, respectivamente), pero no en linfocitos $B$ y niveles muy elevados de IL-1, receptor soluble de interleucina 2 (IL-2R), IL10 y FNT- $\alpha$ en comparación con los casos moderados, que mostraron niveles levemente aumentados o en rangos de normalidad. De igual manera, en casos severos existe una asociación inversamente proporcional entre el recuento de linfocitos $T$ con los niveles de dichas interleucinas. Los autores encontraron que la expresión de interferón gamma por los linfocitos T CD4+ fue más baja en casos severos (aunque no estadísticamente significativo, $p=0.063$ ).

Estos resultados son consistentes con los encontrados por Diao y colaboradores ${ }^{22}$ en 522 pacientes con diagnóstico confirmado de COVID-19 severo. En este estudio los niveles de linfocitos T menores a $800 \mathrm{cel} /$ $\mu \mathrm{L}, \mathrm{CD} 4+$ menores $400 \mathrm{cel} / \mu \mathrm{L}$ y de CD8+ menores a $300 \mathrm{cel} / \mu \mathrm{L}$ se relacionaron negativamente con la supervivencia. Asimismo, los linfocitos T circulantes exhibieron aumento en los marcadores PD-1 y Tim3, que indican un estado de agotamiento, similar al encontrado en otras infecciones crónica virales y en cáncer. ${ }^{23,24}$ En pacientes con neumonía por SARS-CoV-2, este perfil de citoquinas proinflamatorias y depleción linfocitaria, sobre todo en la población de linfocitos T CD8+, predijo la transición entre enfermedad leve y severa. ${ }^{25} \mathrm{En}$ la búsqueda de marcadores tempranos de pronóstico en COVID-19, dos hallazgos pueden ser utilizados en la práctica clínica. El primero es la relación de neutrófilos/ linfocitos T CD8+ mayor a 21.9, la cual mostró un área bajo la curva (AUC) de 0.94 (sensibilidad de $84.6 \%$ y especificidad de $92.6 \%$ ), para predecir casos severos. ${ }^{26}$ El segundo es la relación neutrófilos/linfocitos mayor a 5, que alcanza un AUC de 0.93 (sensibilidad: 84.6\%, especificidad: $96.3 \%$ ) y en mayores de 50 años, un punto de corte de 3.13 o más puede ayudarnos a identificar precozmente los pacientes graves. ${ }^{27}$

Entre los sobrevivientes, el recuento de linfocitos $T$ muestra una cinética peculiar. La linfopenia que acompaña a los casos graves alcanza su pico en la primera semana desde el inicio de los síntomas, su recuperación empieza alrededor de la segunda semana y niveles similares a los casos leves se obtienen a la tercera semana de enfermedad. ${ }^{26}$

Por último, Gong y colaboradores ${ }^{28}$ realizaron un análisis de correlación entre la severidad en COVID-19 y parámetros inflamatorios en 100 pacientes con diagnóstico de neumonía, encontraron puntos de corte de IL-2R mayores a $793.5 \mathrm{U} / \mathrm{mL}(\mathrm{R}=0.564, \mathrm{p}<0.001)$, proteína C reactiva mayor a $30.7 \mathrm{ng} / \mathrm{mL}(\mathrm{R}=0.604, \mathrm{p}$ $<0.001)$ y ferritina mayor a $2,252 \mu \mathrm{g} / \mathrm{L}(\mathrm{R}=0.508, \mathrm{p}<$ 0.001 ) como los de mejor rendimiento.

En síntesis, estos hallazgos demuestran la relación existente entre el síndrome de liberación de citoquinas y la severidad de COVID-19, de tal manera que pueden predecir tempranamente la mortalidad de estos casos, siendo útiles para el escogimiento de aquellos pacien- 
tes que requerirán manejo en cuidados intensivos. Asimismo, se abre la puerta a la utilización de inmunomoduladores, tales como bloqueadores de interleucinas como blanco terapéutico en estos casos.

\section{Terapia inmunomoduladora en COVID-19}

De manera reciente, la campaña para sobrevivir a la sepsis, ${ }^{29}$ en su versión para el manejo de pacientes con COVID-19 severo, se pronunció en contra del uso de esteroides sistémicos en pacientes sin SDRA, también de inmunoglobulina intravenosa, plasma convaleciente y ritonavir/lopinavir; además, consideró evidencia insuficiente para emitir una recomendación respecto al uso de antimaláricos, interferones recombinantes y tocilizumab. El consenso colombiano para el diagnóstico y tratamiento de la infección por SARS-CoV-2 y las directrices mexicanas tampoco contemplan la terapia inmunomoduladora en estos casos. ${ }^{30}$ El tratamiento ideal del síndrome de liberación de citoquinas es desconocido. Sin embargo, la clara relación que hay entre su presentación y la severidad del COVID-19, además de la experiencia acumulada en otros escenarios, abren la posibilidad para la eventual utilización de la terapia inmunomoduladora. Por ejemplo, el uso de terapia antiinterferón gamma en niños con linfohistiocitosis hemofagocítica hereditaria ha demostrado beneficios en la supervivencia y se encuentran en curso estudios clínicos para evaluar su utilidad en niños mayores y adultos. ${ }^{31}$ Anakinra, un antagonista recombinante del receptor de IL-1 mejoró la supervivencia en $73 \%$, en 44 pacientes con LHH secundaria a enfermedades oncológicas, reumáticas e infecciosas ${ }^{32}$ y duplicó la supervivencia de pacientes sépticos con características de síndrome de liberación de citoquinas, en un análisis retrospectivo de un ensayo clínico controlado. ${ }^{33}$ Además del bloqueo de IL-1 e interferón gamma (INF- $\gamma$ ), los niveles elevados de IL-6 vistos en estos pacientes han llevado al uso de tocilizumab, un anticuerpo monoclonal contra el receptor de IL-6 como terapia dirigida contra citoquinas. ${ }^{34,35}$ Asimismo, la inhibición de cinasas Jano, con ruxolitinib, bloquea la señalización emanada de IL-6, INF- $\gamma$ y otras citoquinas. ${ }^{36}$ Esta variedad de medicamentos pueden ser potencialmente benéficos para el tratamiento de pacientes con COVID-19 severo; pero la relación riesgo-beneficio debe ser examinada a la luz de estudios clínicos.

\section{Uso de glucocorticoides}

La evidencia para su uso en COVID-19 es limitada. Estudios observacionales en SARS y MERS no demostraron mejoría en la supervivencia, pero sí un aumento significativo en hiperglucemia, psicosis y necrosis avascular; ${ }^{37}$ mientras que en la neumonía por influenza A $\mathrm{H} 1 \mathrm{~N} 1$, aumentaron las tasas de mortalidad y de infec- ciones secundarias. ${ }^{38}$ En COVID-19, los estudios a la fecha, no soportan su uso rutinario. ${ }^{39}$

\section{Tocilizumab, un inhibidor del receptor IL-6}

Tocilizumab fue evaluado en un estudio retrospectivo de 21 pacientes con COVID-19 severo en cuidados intensivos, a dosis de $400 \mathrm{mg}$ intravenosos al día. Dieciocho pacientes recibieron una sola dosis y tres pacientes requirieron una segunda aplicación por persistencia de fiebre. Setenta y cinco por ciento de los pacientes disminuyeron el requerimiento de oxígeno, se documentó una resolución de las opacidades pulmonares por tomografía en $90.5 \%$, hubo recuperación del recuento linfocitario en $52.6 \%$ de los casos y descenso de la proteína $\mathrm{C}$ reactiva en $84.2 \%$ al quinto día luego de la aplicación de este medicamento. ${ }^{40}$ Con estos resultados, tocilizumab fue incluido en las guías de tratamiento en China. ${ }^{41}$ Al 18 de mayo de 2020, tres estudios se encuentran en curso evaluando la seguridad y eficacia de este medicamento en neumonía por COVID-19: ChiCTR2000029765, ChiCTR2000030796, ChiCTR2000030442 y ChiCTR2000030894.

\section{Inhibidores de JAK}

Estas moléculas son posibles candidatos como terapia contra el COVID-19 severo, dado que son capaces de regular la endocitosis, necesaria para el ingreso del SARS-CoV-2 a la célula a través de AAK1 (proteína quinasa asociada a AP2). ${ }^{42}$ Dentro de este grupo, el baricitinib y ruxolitinib han sido los más estudiados. Sin embargo, su uso genera preocupación por la inhibición de múltiples citoquinas, dentro de ellas el interferón alfa, el cual juega un papel importante en la actividad antiviral. ${ }^{43}$ Estudios con ruxolitinib y jakotinib se encuentran registrados: ChiCTR2000030170 y ChiCTR2000029580.

\section{Antagonista de la familia de IL-1}

Las tres citoquinas más importantes en la familia de IL-1 son la IL-1 beta, la IL-18 e IL-33. Anakinra, un antagonista de IL-1 beta, ha sido usado para el tratamiento de la tormenta de citoquinas inducida por infección, mejorando la supervivencia de los pacientes con sepsis severa. ${ }^{33}$ A la fecha, no existe experiencia de uso en casos severos de COVID-19.

\section{Terapia con plasma convaleciente}

El plasma convaleciente contiene anticuerpos neutralizantes obtenidos de personas recuperadas de ciertos procesos infecciosos. Existe alguna experiencia con la aplicación de plasma convaleciente en influenza A 
H1N1 y SARS-CoV. En estudios observacionales esta terapia y la inmunoglobulina hiperinmune fue asociada con reducción en la mortalidad, en personas que lo recibían en los primeros siete a 10 días de inicio de los síntomas, cuando se encuentra el pico de viremia y la respuesta inmune aún no se ha producido. ${ }^{44,45}$ Recientemente, se publicó la primera serie de casos con cinco pacientes críticamente enfermos con COVID-19 confirmado y SDRA tratados con esta terapia, obteniendo mejoría en su estado clínico. ${ }^{46}$

\section{CONCLUSIONES}

Existe una clara relación entre el síndrome de liberación de citoquinas y la severidad de COVID-19. A la fecha, no se han logrado definir criterios diagnósticos que permitan una identificación temprana de estos casos; sin embargo, la presencia de linfopenia, marcadores de inflamación como niveles elevados de IL-6 y ferritina, así como la relación neutrófilos/linfocitos mayor a 5 , puede ayudar a reconocer precozmente a los pacientes que requerirán cuidado intensivo. Algunos medicamentos inmunomoduladores pueden tener potencial beneficio para el tratamiento de este síndrome, pero se requieren estudios clínicos que avalen su uso y establezcan los riesgos, como la posibilidad de sobreinfección bacteriana y de difícil control de la viremia.

Los autores consideran que las siguientes semanas serán decisivas para definir la utilización ampliada de estos medicamentos y que se hace necesaria una actualización pronta del tema.

\section{REFERENCIAS}

1. La Roseé $\mathrm{P}$, Home AC, Hines $\mathrm{M}$, et al. Recommendations for the management of hemophagocytic lymphohistiocytosis in adults. Blood. 2019:123(33):2465-2477.

2. Valade S, Mariotte E, Azoulay E. Coagulation disorders in hemophagocitic lymphohistiocytosis/macrophage activation syndrome. Crit Care Clin. 2020;36(2):415-426.

3. Yildiz H, Van Den Neste E, Defour JP, Danse E, Yombi JC. Adult haemophagocytic lymphohistiocytosis: a review. QJM. 2020. doi: 10.1093/qjmed/hcaa011.

4. Al-Samkari H, Berliner N. Hemophagocytic lymphohistiocytosis. Annu Rev Pathol Mech Dis. 2018;13:27-49.

5. Du RH, Liang LR, Yang CQ, et al. Predictors of mortality for patients with COVID-19 pneumonia caused by SARS-CoV-2: a prospective cohort study. Eur Respir J. 2020;55(5):2000524. doi: 10.1183/13993003.00524-2020.

6. Wang Z, Yang B, Li Q, Wen L, Zhang R. Clinical features of 69 cases with coronavirus disease 2019 in Wuhan, China. Clin Infect Dis. 2020;71(15):769-777. doi: 10.1093/cid/ciaa272.

7. Ruan Q, Yang K, Wang W, Jiang L, Song J. Clinical predictors of mortality due to COVID-19 based on an analysis of data of 150 patients from Wuhan, China. Intensive Care Med. 2020;46(5):846-848. doi: 10.1007/s00134-020-05991-x.

8. Chen G, Wu D, Guo W, Cao Y, Huang D, Wang H, et al. Clinical and immunological features of severe and moderate coronavirus disease 2019. J Clin Invest. 2020;130(5):2620-2629. doi: 10.1172/JCl137244.

9. Mehta P, McAuley DF, Brown M, Sanchez E, Tattersall RS, Manson JJ; HLH Across Speciality Collaboration, UK. COVID-19: consider cytokine storm syndromes and immunosuppression. Lancet. 2020;395(10229):1033-1034. doi: 10.1016/S01406736(20)30628-0.

10. Cron RQ, Chatham WW. The rheumatologist's role in COVID-19. J Rheumatol. 2020;47(5):639-642. doi: 10.3899/jrheum.200334.

11. Wang D, Hu B, Hu C, Zhu F, Liu X, Zhang J, et al. Clinical Characteristics of 138 Hospitalized Patients With 2019 Novel Coronavirus-Infected Pneumonia in Wuhan, China. JAMA. 2020;323(11):1061-1069. doi: 10.1001/jama.2020.1585.

12. Guan WJ, Ni ZY, Hu Y, Liang WH, Ou CQ, He JX, et al. Clinical characteristics of coronavirus disease 2019 in China. N Engl J Med. 2020;382(18):1708-1720. doi: 10.1056/NEJMoa2002032.

13. Huang $\mathrm{C}$, Wang $\mathrm{Y}$, Li X, et al. Clinical features of patients infected with 2019 novel coronavirus in Wuhan, China. Lancet. 2020;395(10223):497.

14. Zhou F, Yu T, Du R, et al. Clinical course and risk factors for mortality of adult inpatients with COVID-19 in Wuhan, China: a retrospective cohort study. Lancet. 2020;395(10229):1054-1062.

15. Henter JI, Horne A, Arico M, et al. HLH-2004: Diagnostic and therapeutic guidelines for hemophagocytic lymphohistiocytosis. Pediatr Blood Cancer. 2007;48(2):124-131.

16. Fardet L, Galicier L, Lambotte O, et al. Development and validation of the HScore, a score for the diagnosis of reactive hemophagocytic syndrome. Arthritis Rheumatol. 2014;66(9):2613-2620.

17. Eloseily EMA, Minoia F, Crayne CB, Beukelman T, Ravelli $A$, Cron RQ. Ferritin to erythrocyte sedimentation rate ratio: simple measure to identify macrophage activation syndrome in systemic juvenile idiopathic arthritis. ACR Open Rheumatol. 2019;1(6):345-349.

18. Wong CK, Lam CWK, Wu AKL, et al. Plasma inflammatory cytokines and chemokines in severe acute respiratory syndrome. Clin Exp Immunol. 2004;136:95-103.

19. Mahallawi WH, Khabour OF, Zhang Q, Makhdoum HM, Suliman BA. MERS-CoV infection in humans is associated with a pro-inflammatory Th1 and Th17 cytokine profile. Cytokine. 2018;104:8-13.

20. Liu Q, Zhou Y, Yang Z. The cytokine storm of severe influenza and development of immunomodulatory therapy. Cell Mol Immunol. 2016;13(1):3-10.

21. National Health Commission of the People's Republic of China. Protocol for prevention and control of COVID-19 (edition 6). China CDC Wkly [Internet]. 2020;2(19):321-326. Available in: https://www.chinacdc.cn/en/COVID19/202003/ P020200323390496137554.pdf

22. Diao B, Wang Ch, Tan $\mathrm{Y}$, et al. Reduction and functional exhaustion of $\mathrm{T}$ cells in patient with coronavirus disease 2019 (COVID-19). MedRxiv. 2020. Available in: https://doi. org/10.1101/2020.02.18.20024364

23. Ng CT, Snell LM, Brooks DG, Oldstone MB. Networking at the level of host immunity: immune cell interactions during persistent viral infections. Cell Host Microbe. 2013;13(6):652-664.

24. Fenwick $C$, Joo V, Jacquier $P$, et al. T-cell exhaustion in HIV infection. Immunol Rev. 2019;292(1):149-163.

25. Wan S, Yi Q, Fan S, et al. Characteristics of lymphocyte subsets and cytokines in peripheral blood of 123 hospitalized patients with 2019 novel coronavirus pneumonia (NCP). MedRxiv. 2020. Available in: https://doi.org/10.1101/2020.02.10.20021832

26. Liu J, Li S, Liu J, et al. Longitudinal characteristics of lymphocyte responses and cytokine profiles in the peripheral blood of SARSCoV-2 infected patients. MedRxiv. 2020. Available in: https://doi. org/10.1101/2020.02.16.20023671

27. Liu J, Liu Y, Xang $P$, et al. Neutrophil-to-lymphocyte ratio predicts severe illness patients with 2019 novel coronavirus in the early stage. MedRxiv. 2020. Available in: https://doi. org/10.1101/2020.02.10.20021584

28. Gong J, Dong H, Xia Q, et al. Correlation analysis between disease severity and inflammation-related parameters in patients with COVID-19 pneumonia. MedRxiv. 2020. Available in: https:// doi.org/10.1101/2020.02.25.20025643

29. Alhazzani W, Maller MH, Arabi YM, et al. Surviving Sepsis Campaign: guidelines on the management of critically ill adults with Coronavirus Disease 2019 (COVID-19). Intensive Care Med. 2020;46(5):854-887. doi: 10.1007/s00134-020-06022-5. 
30. Grupo ACIN- IETS de Consenso Colombiano para recomendaciones de atención COVID19. Consenso colombiano de atención, diagnóstico y manejo de la infección por SARSCOV-2/COVID-19 en establecimientos de atención de la salud. Infectio. 2020;24(3):S1.

31. Prencipe G, Bracaglia C, Caiello I, et al. The interferon-gamma pathway is selectively up-regulated in the liver of patients with secondary hemophagocytic lymphohistiocytosis. PLoS One. 2019;14(12):e0226043.

32. Eloseily EM, Weiser P, Crayne CB, et al. Benefit of anakinra in treating pediatric secondary hemophagocytic lymphohistiocytosis. Arthritis Rheumatol. 2020;72(2):326-334.

33. Shakoory B, Carcillo JA, Chatham WW, et al. Interleukin-1 receptor blockade is associated with reduced mortality in sepsis patients with features of macrophage activation syndrome: reanalysis of a prior phase III trial. Crit Care Med. 2016;44:275-281.

34. Luo P, Liu Y, Qiu L, Liu X, Liu D, Li J. Tocilizumab treatment in COVID-19: A single center experience. J Med Virol. 2020;92(7):814-818. doi: 10.1002/jmv.25801.

35. Michot JM, Albiges L, Chaput N, et al. Tocilizumab, an anti-IL-6 receptor antibody, to treat COVID-19-related respiratory failure: a case report. Ann Oncol. 2020;31(7):961-964. doi: 10.1016/j. annonc.2020.03.300.

36. Crayne CB, Albeituni S, Nichols KE, Cron RQ. The immunology of macrophage activation syndrome. Front Immunol. 2019;10:119.

37. Arabi YM, Mandourah Y, Al-Hameed F, et al; Saudi Critical Care Trial Group. Corticosteroid therapy for critically ill patients with Middle East respiratory syndrome. Am J Respir Crit Care Med. 2018;197(6):757-767

38. Ni YN, Chen G, Sun J, Liang BM, Liang ZA. The effect of corticosteroids on mortality of patients with influenza pneumonia: a systematic review and meta-analysis. Crit Care. 2019;23(1):99.

39. Russell CD, Millar JE, Baillie JK. Clinical evidence does not support corticosteroid treatment for 2019-nCoV lung injury. Lancet. 2020;395(10223):473-475.
40. Xu X, Han M, Li T, et al. Effective treatment of severe COVID-19 patients with tocilizumab. ChinaXiv. 2020. doi: $10.12074 / 202003.00026$.

41. Diagnosis and treatment protocol for novel coronavirus pneumonia (Trial Version 7). Chin Med J (Engl). 2020;133(9):1087-1095.

42. Sanders JM, Monogue ML, Jodlowski TZ, Cutrell JB. Pharmacologic treatments for coronavirus disease 2019 (COVID-19): a review. JAMA. 2020;323(18):1824-1836. doi: 10.1001/jama.2020.6019.

43. Zhang $W$, Zhao $Y$, Zhang $F$, et al. The use of anti-inflammatory drugs in the treatment of people with severe coronavirus disease 2019 (COVID-19): the perspectives of clinical immunologists from China. Clin Immunol. 2020;214:108393. doi: 10.1016/j. clim.2020.108393.

44. Hung IF, To KK, Lee CK, et al. Convalescent plasma treatment reduce mortality in patients with severe pandemic influenza $A$ (H1N1) 2009 virus infection. Clin Infect Dis. 2011;52(4):447-456.

45. Mair-Jenkins J, Saavedra-Campos M, Baillie JK, et al; Convalescent Plasma Study Group. The effectiveness of convalescent plasma and hyperimmune immunoglobulin for the treatment of severe acute respiratory infections of viral etiology: a systematic review and exploratorymeta-analysis. J Infect Dis. 2015;211(1):80-90.

46. Shen C, Wang Z, Zhao F, et al. Treatment of 5 critically ill patients with COVID-19 with convalescent plasma. JAMA. 2020;323(16):1582-1589. doi: 10.1001/jama.2020.4783.

Correspondencia:

Mario Andrés Hernández-Sómerson

E-mail: somerson18@hotmail.com 